VIII Congresso Brasileiro de Informática na Educação (CBIE 2019)

Anais do XXV Workshop de Informática na Escola (WIE 2019)

\title{
EcosAR: simulador de ecossistemas utilizando realidade aumentada
}

\section{Rodrigo Wernke Pereira ${ }^{1}$, Dalton Solano dos Reis ${ }^{1}$, Roberta Andressa Pereira ${ }^{2}$, Mauricio Capobianco Lopes ${ }^{3}$}

\author{
${ }^{1}$ Departamento de Sistemas e Computação \\ Universidade Regional de Blumenau (FURB) - Rua Antonio da Veiga, 140 - CEP 89.030-903 - \\ Blumenau, SC - Brasil \\ ${ }^{2}$ Departamento de Ciências Naturais \\ Universidade Regional de Blumenau (FURB) - Blumenau, SC - Brasil \\ ${ }^{3}$ Programa de Pós-Graduação em Ensino de Ciências Naturais e Matemática \\ Universidade Regional de Blumenau (FURB) - Blumenau, SC - Brasil \\ rodrigowernkedfurb.br, daltondfurb.br, rapereira@furb.br, mclopesefurb.br
}

\begin{abstract}
This paper presents EcosAR, an application that aims to simulate ecosystems using the technologies of Augmented Reality and Tangible User Interfaces. The activity of simulating ecosystems using these technologies enables the user to observe and control natural phenomena such as water cycle, tree life cycle, wind and temperature. The application was developed using the Unity graphics engine and the Vuforia library. Functional tests were carried out as well as an activity with academics from the Biological Sciences course, who answered a survey. The results indicated the feasibility of applying these technologies in the simulation of ecosystems, as well as identified possibilities of improvements in the application.
\end{abstract}

Resumo: Este artigo apresenta o EcosAR, aplicativo que tem como objetivo simular ecossistemas utilizando as tecnologias de Realidade Aumentada e Interfaces de Usuário Tangivel. A atividade de simular ecossistemas utilizando estas tecnologias possibilita ao usuário a observação e controle de fenômenos naturais como o ciclo da água, o ciclo de vida das árvores, vento e temperatura. O aplicativo foi desenvolvido utilizando o motor gráfico Unity e com a biblioteca Vuforia. Foram realizados testes de funcionalidade e com acadêmicos do curso de Ciências Biológicas, os quais responderam um questionário. Os resultados indicaram a viabilidade de aplicação dessas tecnologias na simulação de ecossistemas, bem como identificaram possibilidades de melhorias no aplicativo.

\section{Introdução}

O uso da tecnologia cria transformações, incluindo a área de educação, pois ela promove novas formas de adquirir e transmitir o conhecimento. Desta forma, faz-se necessário pensar as diversas maneiras de usar o potencial que a tecnologia oferece para auxiliar no ensino e aprendizagem dos conteúdos, em uma era na qual ela se encontra cada vez mais presente na vida das pessoas. Atualmente, com o advento da tecnologia, existem várias formas de ensinar sobre o meio ambiente. Um dos temas abordados no ensino fundamental são os ecossistemas.

A palavra ecossistema refere-se a um conjunto de organismos vivos que interagem não só com o meio físico que os rodeia, mas também com a química ambiental e com o meio social e biológico em que estão inseridos (...) (CARAPETO, 2016, p. $15)$. 
VIII Congresso Brasileiro de Informática na Educação (CBIE 2019)

Anais do XXV Workshop de Informática na Escola (WIE 2019)

Ricklefs (2010, p. 4) afirma que ecossistemas são sistemas ecológicos complexos e grandes, às vezes incluindo muitos milhares de diferentes tipos de organismos, vivendo cada um em uma grande variedade de meios. Pode-se pensar em um ecossistema como um organismo que possui processos internos e troca com os arredores externos (RICKLEFS, 2010, p. 4). Segundo Odum (1988, p.13), chama-se ecossistema qualquer unidade (biossistema) que abranja todos os organismos funcionando em conjunto, interagindo com o ambiente físico de modo que o fluxo de energia produza estruturas bióticas claramente definidas e uma ciclagem de materiais entre as partes vivas e não vivas. Os ecossistemas são caracterizados pela magnitude de processos ecológicos fundamentais, como a produção e a ciclagem de materiais, de acordo com Odum (1972 apud COELHO; MOTTA, 2009, p.70).

Fiedler et al. (1997 apud VIGLIO; FERREIRA, 2013, p. 3) relatam que ecossistemas são unidades autorreguladas que seguem uma trajetória linear de desenvolvimento em direção a uma particular diversidade biológica e um estado de estabilidade denominado clímax. Com esta perspectiva, são considerados muito mais como eventos externos do que propriedades intrínsecas dos sistemas ecológicos. O paradigma de equilíbrio é uma das ideias mais antigas e dominantes na ecologia e foi fundamental no desenvolvimento do conceito de ecossistema, segundo Pickett et al. (1992 apud VIGLIO; FERREIRA, 2013, p. 4).

Os ecossistemas podem ser ensinados ou aprendidos com base no uso de simuladores com os quais é possível imitar ou reproduzir situações reais ou mesmo propostas de forma abstrata dos fenômenos a serem simulados (GREIS; REATEGUI, 2010). Os experimentos que utilizam estas possibilidades buscam entender o comportamento ou avaliar estratégias para a sua operação, segundo Aldrich (2009). Segundo Greis e Reategui (2010), as vantagens de trabalhar com modelos simulados por computador no campo educacional são muitas, desde a oportunidade de tornar possível a reprodução de processos muito lentos ou muito perigosos para serem reproduzidos no ambiente natural para a observação dos fenômenos, até mesmo pela redução dos custos envolvidos no projeto. De acordo com Torga (2007, p. 43), simulação é a importação da realidade para um ambiente controlado no qual se pode estudar o comportamento do mesmo, sob diversas condições, sem riscos físicos ou grandes custos envolvidos. A simulação é o ato de imitar um procedimento real em menor tempo e com um custo menor, possibilitando um melhor estudo do que vai acontecer e de como consertar erros que gerariam grandes gastos (O'KANE; TAYLOR; SPENCELEY, 2000).

Uma das áreas que vem auxiliando o desenvolvimento de simuladores é a Realidade Aumentada (RA). RA é definida por Kirner et al. (2006) como uma técnica para conectar o ambiente virtual ao ambiente real do usuário, proporcionando uma interação natural, sem necessidade de treinamento ou adaptação. Diferente da Realidade Virtual, na qual o usuário é imerso em um ambiente criado digitalmente, a realidade aumentada combina o mundo real com o mundo virtual, no qual estes dois coexistem alinhados e em tempo real (AZUMA, 2001 apud ROMÃO; GONÇALVES, 2013, p. 23). A realidade aumentada não cria mundos virtuais, mas maximiza elementos do mundo real para que se possa melhorar a interação e perceber aspectos sensoriais imperceptíveis nas dimensões reais (FRANÇA; SILVA, 2017, p. 3). De acordo com Kirner e Siscoutto (2007, p. 5), o fato dos objetos virtuais serem trazidos para o espaço físico do usuário por sobreposição permitiu interações tangíveis mais fáceis e naturais, sem o uso de equipamentos especiais.

A Realidade Aumentada permite explorar a Interface de Usuário Tangível. Segundo Jetter (2013 apud REIS; GONÇALVES, 2016, p. 95) interfaces podem ser entendidas como uma camada de comunicação entre dois elementos: um usuário que emite comandos e um artefato ou sistema que responde a esses comandos, promovendo, assim, uma interação. Ullmer e Ishii (2001) definem que Interfaces de Usuário Tangíveis são representações físicas para dados digitais, permitindo que objetos físicos tenham controle no mundo virtual. Diferente de teclados e mouses, as formas e posições físicas dos objetos de IUT são um importante papel no mundo virtual. Diante deste 
contexto, este trabalho apresenta um aplicativo de simulação de ecossistemas, com base em RA para visualização e o uso de IUT para o usuário manipular características simuladas da cena.

Ressalta-se que a pesquisa de trabalhos correlatos, no SBIE, retornou uma única publicação sobre o ensino de ecossistemas, o trabalho de Ferreira et al. (2018) que destaca um jogo que simula um sonho de um garoto que deve manter o equilíbrio de um sistema interagindo com ele. As tecnologias empregadas e o tipo de elementos simulados são diferentes do presente artigo.

Nas seções a seguir serão destacados: o método, o funcionamento do aplicativo, os resultados e as conclusões.

\section{Método}

O método para produção e avaliação do aplicativo foi delineado do seguinte modo:

a) estudo: nessa etapa foram aprofundados os conceitos sobre ecossistemas e RA e IUT;

b) especificação: nessa etapa foi realizada a definição sobre quais seriam os elementos dos ecossistemas a serem simulados, bem como foi feito o detalhamento da estrutura de análise e projeto do aplicativo, seguindo as etapas da engenharia de software;

c) implementação: essa etapa consistiu em escrever computacionalmente os códigos relativos ao aplicativo especificado;

d) testes funcionais: nessa etapa foram feitos testes para garantir o adequado funcionamento de todas as rotinas implementadas de acordo com a especificação;

e) testes de usabilidade: nessa etapa, o simulador foi submetido à testes por estudantes do curso de Ciências Biológicas para verificar sua eficácia;

f) avaliação: nessa etapa foram analisados os resultados obtidos com o uso do aplicativo pelo grupo de usuários.

Para a avaliação proposta na etapa (e) foi produzido um questionário e foram realizadas observações sobre o comportamento dos estudantes participantes.

\section{Descrição do Aplicativo}

Para o desenvolvimento do aplicativo foram utilizados o motor gráfico Unity, em conjunto com a biblioteca Vuforia, e o ambiente de desenvolvimento Visual Studio 2017. Para a geração dos marcadores foram utilizadas as ferramentas Photoshop CC 2019 e AR Marker Generator (BROSVISION, 2019) que gera imagens aleatórias e otimizadas para a interpretação de marcadores com Realidade Aumentada. Para a modelagem dos objetos foi utilizado a ferramenta Blender.

O aplicativo desenvolvido disponibiliza para o usuário uma forma de simular comportamentos de um ecossistema, concedendo o controle de elementos que existem na natureza, como o vento e a temperatura.

A Figura 1 apresenta os possíveis comportamentos que podem ser realizados dentro do aplicativo, sendo que os paralelogramos são os elementos que o usuário pode controlar, os losangos são as condições necessárias para cada processo e os retângulos correspondem aos resultados obtidos através dos controles realizados. 
VIII Congresso Brasileiro de Informática na Educação (CBIE 2019)

Anais do XXV Workshop de Informática na Escola (WIE 2019)

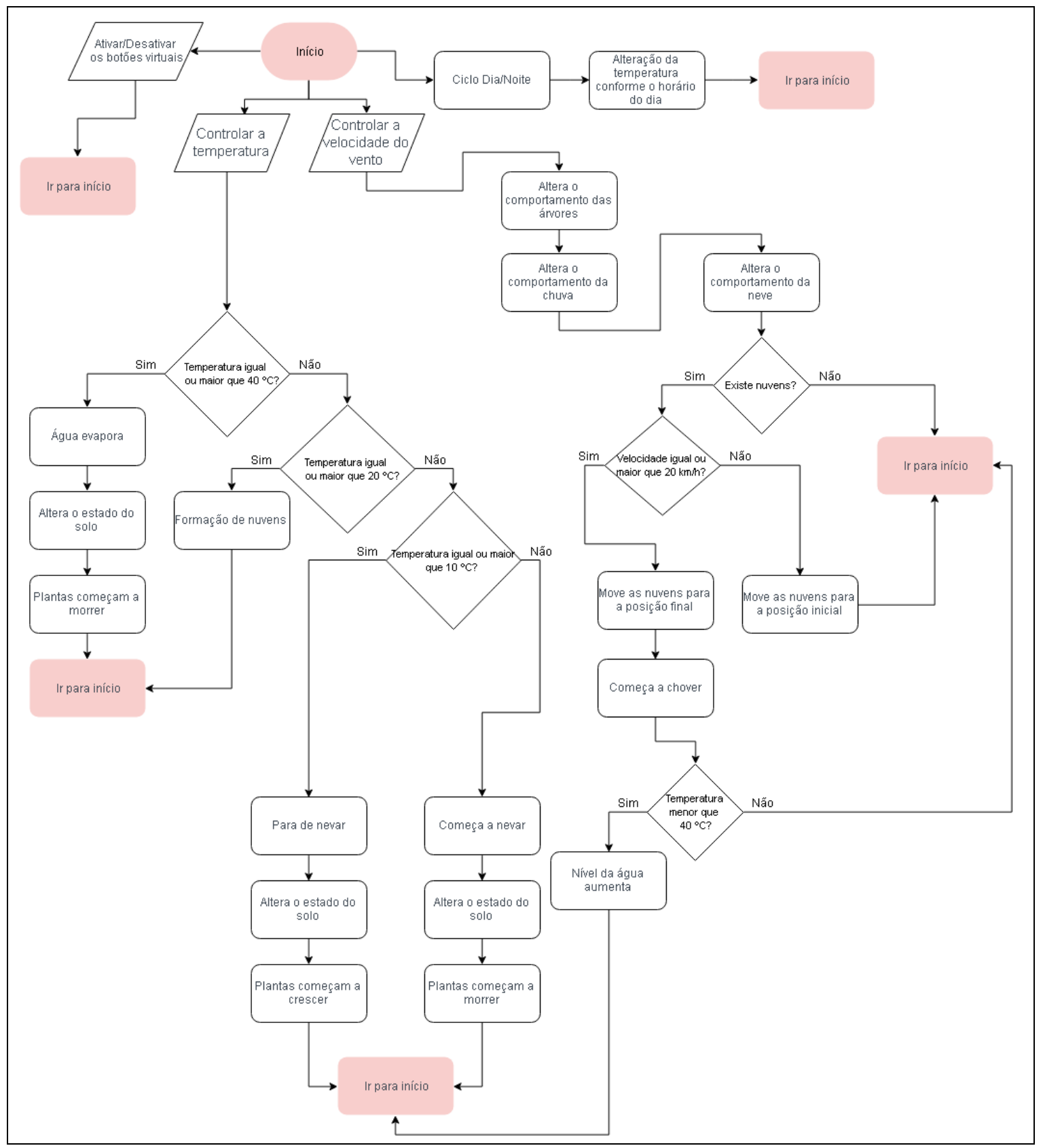

Fonte: elaborado pelos autores.

Figura 1 - Fluxograma de comportamentos do aplicativo

Como pode ser visto na Figura 1, a simulação realiza diferentes comportamentos para diferentes interações do usuário, sendo que mais de um comportamento pode ocorrer ao mesmo tempo, assim gerando um ambiente em que várias simulações ocorrem simultaneamente. Fora do controle do usuário ocorre o ciclo de dia e noite, fazendo com que a temperatura da simulação seja alterada conforme o horário, sendo que durante o dia a simulação aumenta quatro graus e durante a noite a temperatura diminui quatro graus. Quando o usuário altera a temperatura com o uso do marcador, o valor de temperatura calculado pelo ciclo é ignorado e o valor escolhido pelo usuário é utilizado.

O controle do usuário é feito com o uso da câmera do dispositivo móvel em conjunto com marcadores para visualizar a aplicação. Estes marcadores, conforme apresentado na Figura 2, são formados por imagens que são reconhecidas e interpretadas pelo aplicativo, fazendo possível o 
VIII Congresso Brasileiro de Informática na Educação (CBIE 2019)

Anais do XXV Workshop de Informática na Escola (WIE 2019)

desenho da cena sobre o marcador específico. Desta forma, o usuário é capaz de interagir com a aplicação utilizando Realidade Aumentada e Interfaces de Usuário Tangível, rotacionando os marcadores Vento e Temperatura para controlar os elementos que são visualizados sobre o marcador Cena e simular diversos comportamentos.
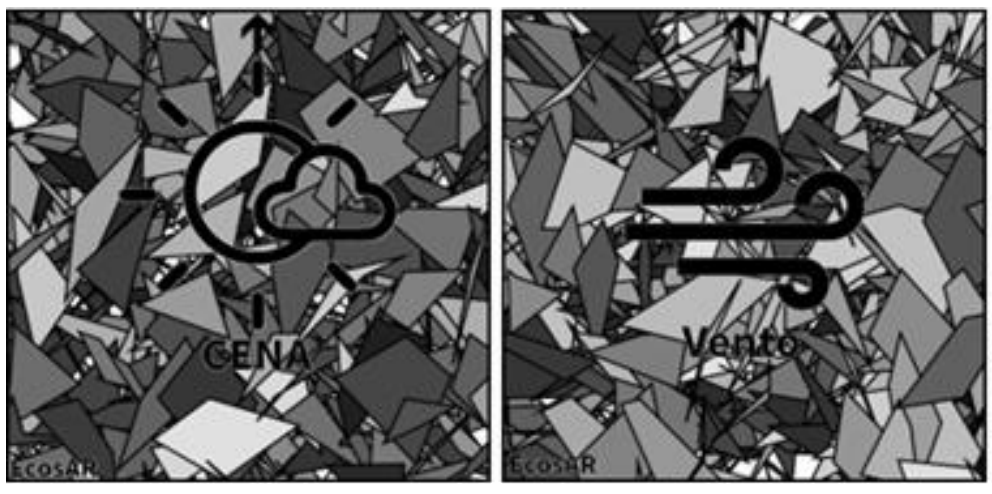

elaborado pelos autores.

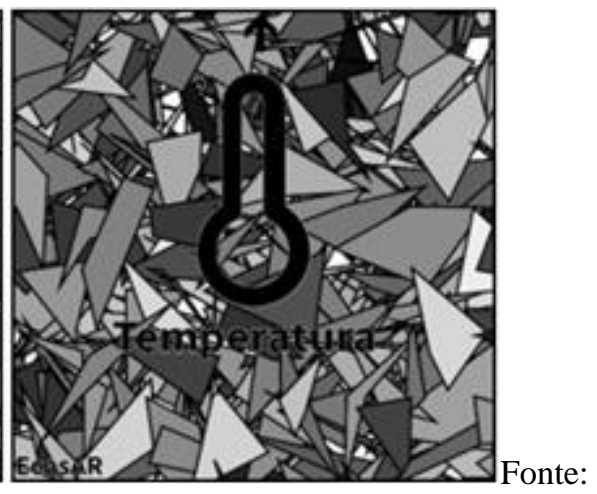

Fonte:

Figura 2- Marcadores da aplicação

A aplicação tem a tela menu inicial e a tela na qual ocorre a simulação. A tela menu inicial, como pode ser vista na Figura 3, é composta por três botões que servem para iniciar a simulação, conhecer sobre a origem do aplicativo e sair da aplicação.

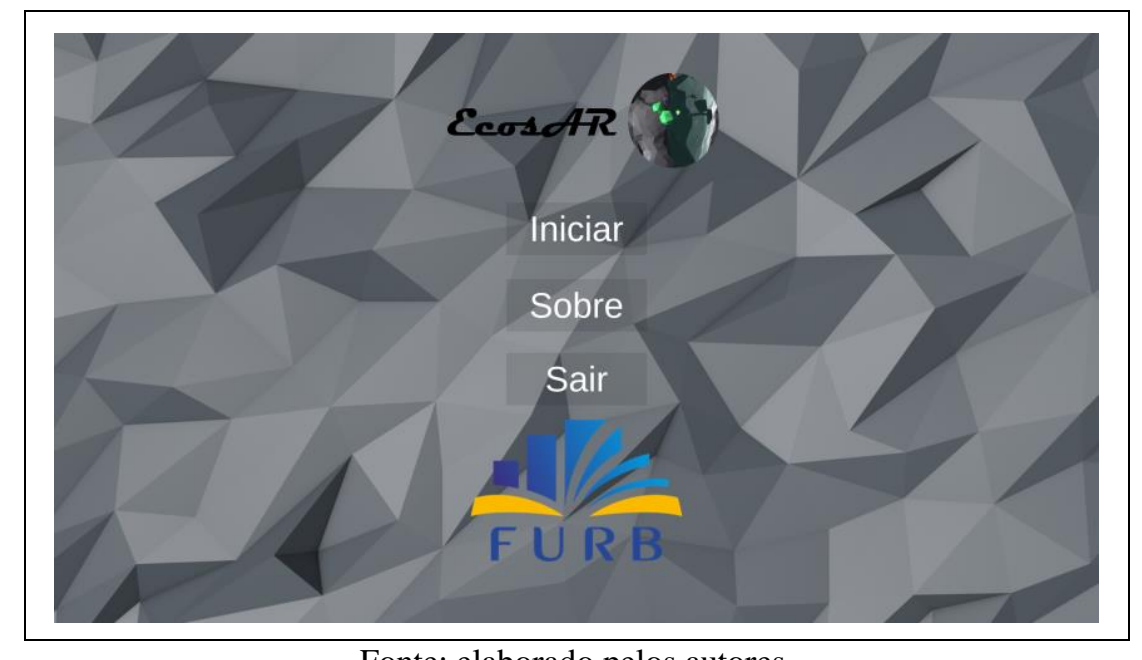

Fonte: elaborado pelos autores.

Figura 3 - Menu do aplicativo

Ao clicar no botão Iniciar o usuário deve mirar a câmera do dispositivo móvel em direção ao marcador cena, com isso é possível visualizar a simulação. Ela começa com a temperatura em zero graus e com a velocidade do vento em zero quilômetros por hora. A partir deste ponto, o usuário pode começar a interagir com a cena utilizando os marcadores. Como citado anteriormente, dependendo da alteração do usuário, diferentes propriedades da simulação podem mudar. A Figura 4 mostra a simulação ao ser iniciada e a descrição de cada componente disponível. 
VIII Congresso Brasileiro de Informática na Educação (CBIE 2019)

Anais do XXV Workshop de Informática na Escola (WIE 2019)

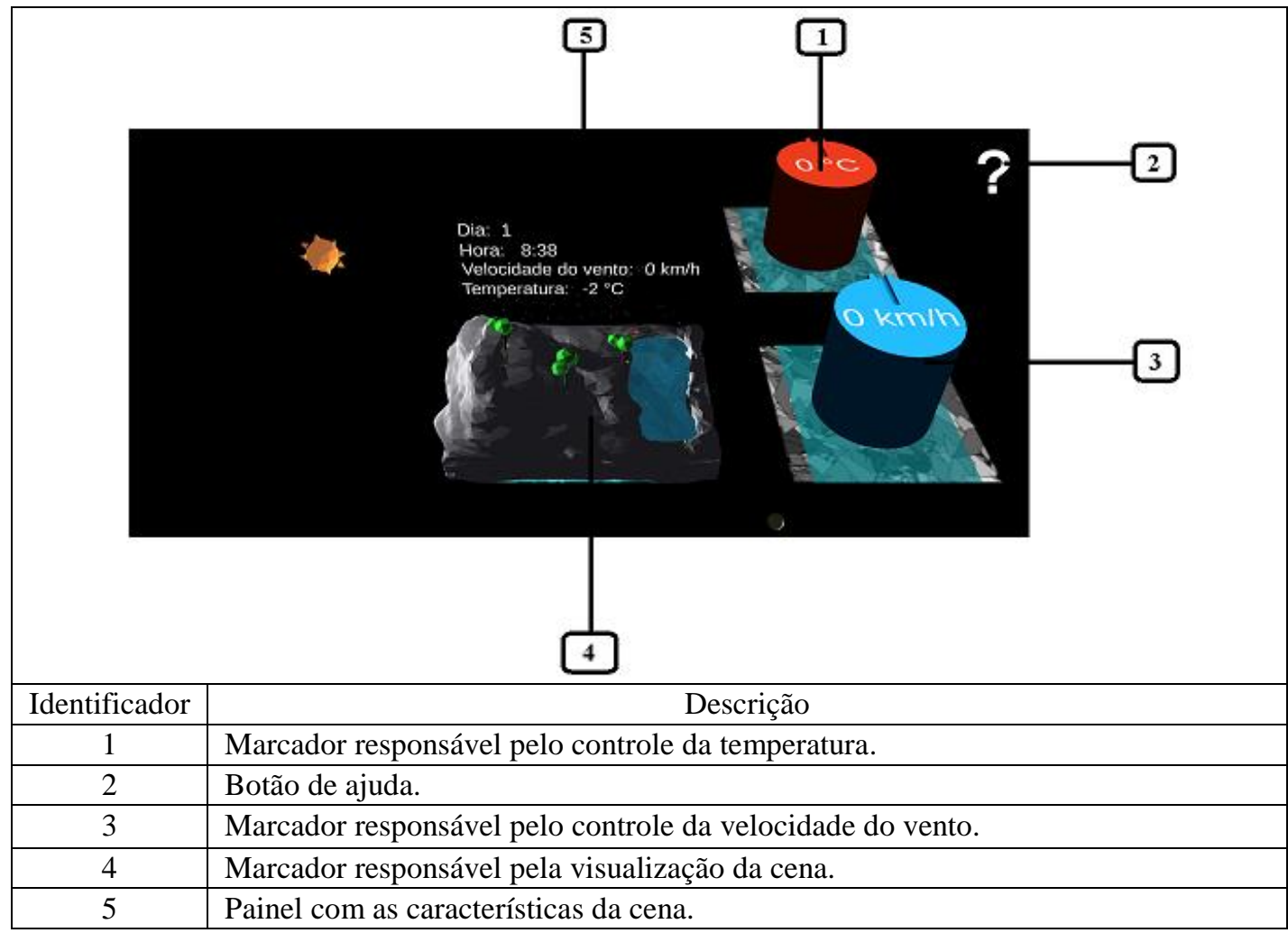

Fonte: elaborado pelos autores.

\section{Figura 4 - Início da simulação}

Durante a simulação o usuário pode observar um painel com as características atuais da cena (Figura 5). Nele é possível observar em qual dia a simulação se encontra (item 1), a hora atual da simulação (item 2), a velocidade do vento na simulação (item 3) e a temperatura da simulação (item 4).

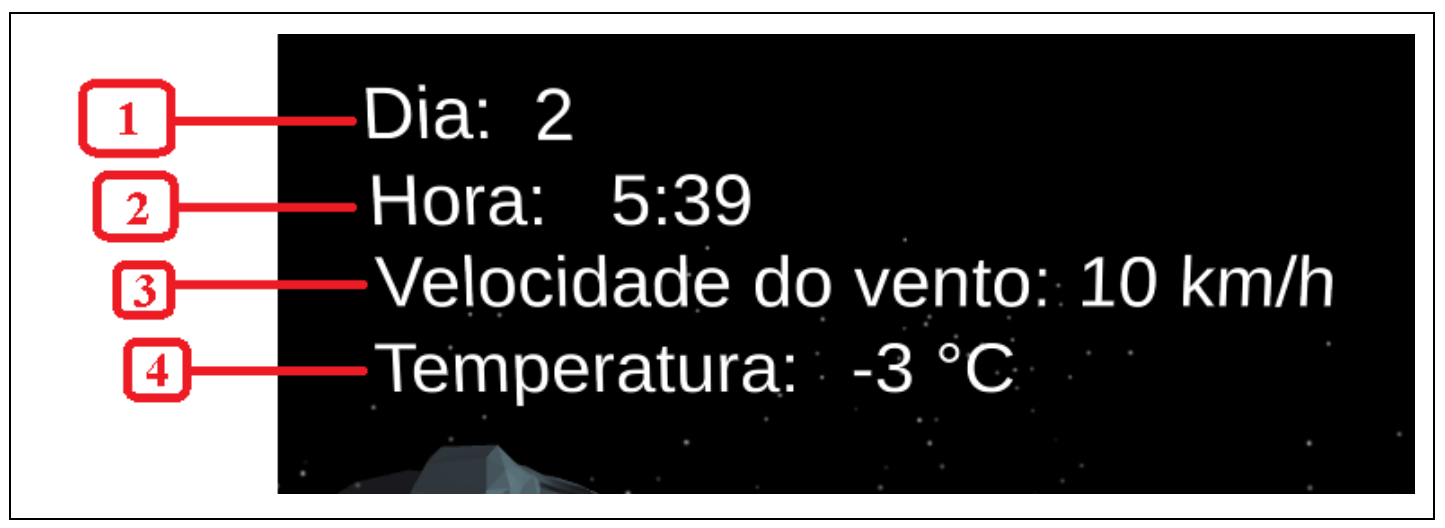

Fonte: elaborado pelos autores.

Figura 5 - Painel da simulação

Com base nesses elementos, o usuário realiza as simulações que deseja e pode verificar os efeitos das variáveis intervenientes no ecossistema. 
VIII Congresso Brasileiro de Informática na Educação (CBIE 2019)

Anais do XXV Workshop de Informática na Escola (WIE 2019)

\section{Análise dos Resultados}

Este capítulo apresenta os testes realizados com o aplicativo. São apresentadas duas seções: uma tratando dos testes de funcionalidade e outra abordando os testes com acadêmicos do curso de Ciências Biológicas.

\subsection{Testes de Funcionalidade}

Para validar as funcionalidades do aplicativo foram realizados testes na plataforma Android. Nesta plataforma todas as funcionalidades se comportaram conforme o esperado. Durante o processo de desenvolvimento do trabalho diversos testes foram executados para validar o comportamento de vários componentes da cena. Estes testes levaram em consideração a visualização e as alterações que o usuário fazia com os marcadores.

No decorrer do desenvolvimento do trabalho foi notado um problema de sensibilidade nos marcadores, a qual era muita alta, dificultando a usabilidade do aplicativo. Com isso, foi adicionado um algoritmo para diminuir a sensibilidade dos marcadores e facilitar o seu uso. Outra melhoria desenvolvida foram os textos de ajuda ao usuário. Foi percebido que seria mais fácil a visualização dos mesmo sem a utilização de Realidade Aumentada, pois os usuários já estariam acostumados com o estilo.

Também foi observado, durante os testes, que o material utilizado na impressão dos marcadores poderia afetar o uso da aplicação, sendo que materiais que refletem a luz com mais intensidade podem dificultar o reconhecimento dos marcadores pelo aplicativo. Outro fator importante foi a qualidade da câmera do dispositivo móvel, que também pode afetar a identificação dos marcadores. Durante a utilização do aplicativo notou-se o alto consumo da bateria do dispositivo móvel, devido ao fato do aplicativo utilizar a câmera constantemente e ser uma aplicação gráfica, consequentemente aumentando o consumo de energia.

\subsection{Testes de Utilização com Acadêmicos do Curso de Ciências Biológicas}

Nos testes realizados com usuários estavam presentes oito alunos do curso de Ciências Biológicas da instituição. Fotos dos testes realizados podem ser visualizadas na Figura 6.

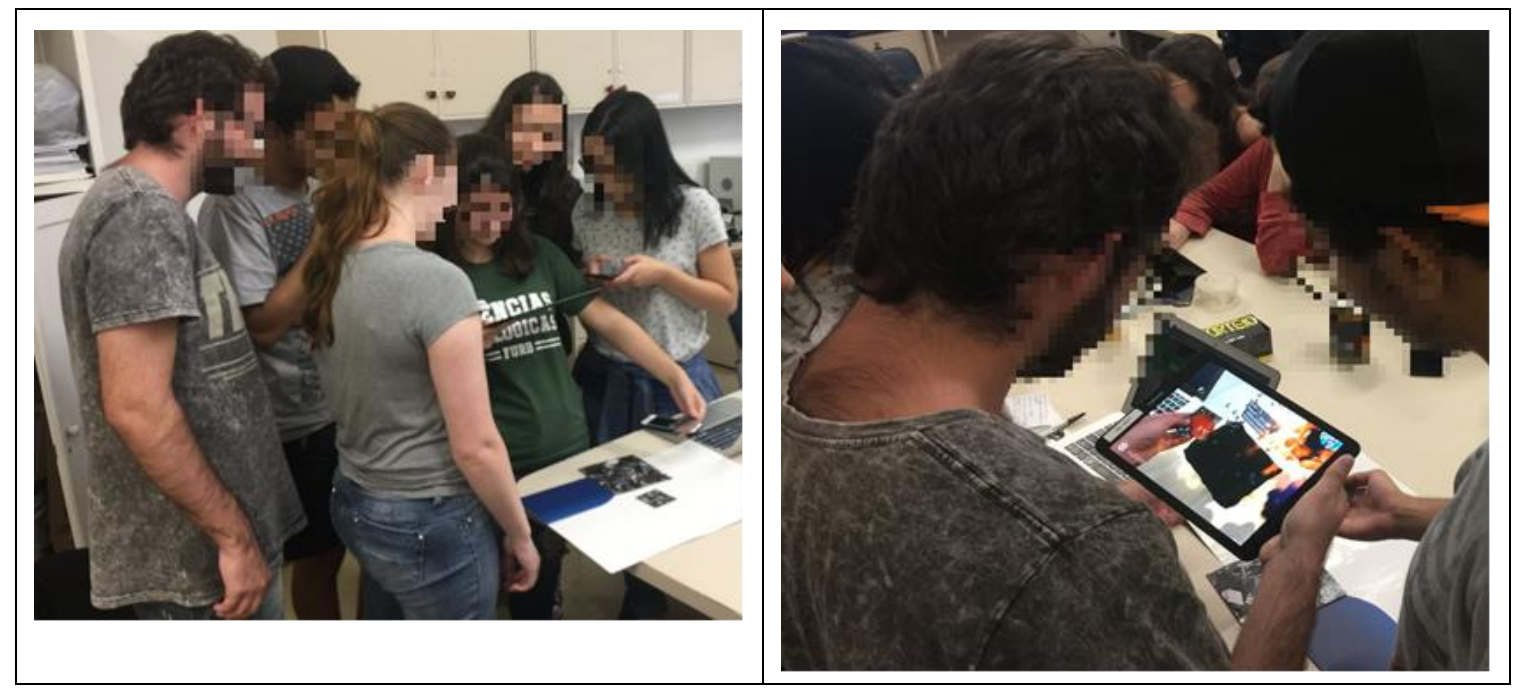

Fonte: elaborado pelos autores.

Figura 6 - Acadêmicos testando a aplicação 
VIII Congresso Brasileiro de Informática na Educação (CBIE 2019)

Anais do XXV Workshop de Informática na Escola (WIE 2019)

Foram distribuídos os marcadores do trabalho desenvolvido para os alunos junto com um questionário online para testar a aplicação. A primeira etapa do questionário consiste em recolher informações sobre o perfil dos usuários cujas respostas estão disponibilizadas na Tabela 1.

Tabela 1 - Perfil dos entrevistados

\begin{tabular}{c|c|c}
\hline Sexo & Feminino & $77,8 \%$ \\
& Masculino & $22,2 \%$ \\
\hline Idade & 18 & $11,1 \%$ \\
& 19 & $11,1 \%$ \\
& 20 & $44,4 \%$ \\
& 21 & $11,1 \%$ \\
& 22 & $11,1 \%$ \\
& 55 & $11,1 \%$ \\
\hline Grau de escolaridade & Ensino superior incompleto & $88,9 \%$ \\
& Ensino superior completo & $11,1 \%$ \\
\hline Utiliza dispositivos móveis & Frequentemente & $100 \%$ \\
com frequência & \multicolumn{2}{|c}{ Sim } \\
\hline Já utilizou aplicações com & Não & $55,6 \%$ \\
Realidade Aumentada & \multicolumn{2}{c}{$44,4 \%$} \\
\hline
\end{tabular}

Como pode ser observado, a maior parte dos alunos possuíam entre 18 e 22 anos, sendo a maioria do sexo feminino. Todos utilizavam dispositivos móveis com frequência e mais da metade dos entrevistados já utilizou uma aplicação com Realidade Aumentada.

A segunda etapa do questionário trazia o passo a passo que demonstrava todas as funcionalidades básicas do aplicativo, como o controle de temperatura e velocidade do vento, os quais todos os entrevistados conseguiram concluir. A terceira parte do questionário foi utilizada para obter a opinião dos entrevistados em relação à usabilidade de manipulação da cena do aplicativo, a usabilidade em geral do aplicativo e se o aplicativo cumpriu seu objetivo. A Tabela 2 mostra os resultados da terceira parte do questionário, sendo as respostas destas perguntas valores entre um e cinco.

Tabela 2 - Opinião dos entrevistados sobre o aplicativo

\begin{tabular}{c|l|l}
\hline Usabilidade de manipular a & 3 & $11,1 \%$ \\
cena & 4 & $33,3 \%$ \\
& 5 & $55,6 \%$ \\
\hline Usabilidade do aplicativo em & 3 & $11,1 \%$ \\
geral & 5 & $88,9 \%$ \\
\hline Cumpriu seu objetivo de & 2 & $11,1 \%$ \\
desenvolver um simulador de & 4 & $11,1 \%$ \\
ecossistemas para & 5 & $77,8 \%$ \\
dispositivos móveis & \\
\hline
\end{tabular}

Os resultados foram considerados bons visto que a grande maioria dos entrevistados demonstrou interesse pelo aplicativo e pelo seu meio de manipulação e controle da simulação. No final do questionário foram adicionadas críticas e sugestões sobre a aplicação as quais podem se tornar propostas para a continuação do trabalho, entre os quais destacam-se: adicionar animais na simulação; incrementar a quantidade de terrenos possíveis; simular outras características do clima; aumentar a variedade de plantas na simulação; incluir novos meios de controle com IUT; implementar controles para a duração do dia. 
VIII Congresso Brasileiro de Informática na Educação (CBIE 2019)

Anais do XXV Workshop de Informática na Escola (WIE 2019)

\section{Conclusões}

Diante dos resultados apresentados o aplicativo provou ser capaz de simular ecossistemas com dispositivos móveis utilizando Realidade Aumentada e Interface de Usuário Tangível. O aplicativo desenvolvido mostrou-se intuitivo e responsivo, com suas funcionalidades operando conforme o esperado. O objetivo de desenvolver um simulador de ecossistemas para dispositivos móveis foi atingido e comprovado com a realização dos testes. Mesmo sendo realizados com um grupo pequeno de alunos, foi possível obter resultados satisfatórios. O aplicativo tem o potencial de auxiliar o ensino de conceitos como ciclo e ecossistemas.

\section{Agradecimentos}

Agradecemos à Fundação de Apoio à Pesquisa e Inovação do Estado de Santa Catarina (FAPESC) pelo financiamento ao (LIFE) da (FURB) e à FURB pelas condições oferecidas para a realização deste trabalho.

\section{Referências}

ALDRICH, Clark. The complete guide to simulations and serious games. San Francisco: Pfeiffer, 2009. 576 p.

BROSVISION. Augmented Reality Marker Generator. 2019. Disponível em: $<\mathrm{http}: / /$ www.brosvision.com/ar-marker-generator/>. Acesso em: 1 jun.2019.

CARAPETO, Cristina. Ecossistemas de Transição. São Paulo: Leya, 2016. 128 p.

COELHO, Pinto; MOTTA, Ricardo. Fundamentos em ecologia. [s. L.]: Artmed Editora, 2009. 252 p.

FERREIRA, T. S. D. et al. AdventureSECO: Jogo Educacional para o Ensino de Conceitos sobre Ecossistemas de Software. In: SIMPÓSIO BRASILEIRO DE INFORMÁTICA NA EDUCAÇÃO, 2018. Proceedings.... Fortaleza(CE): SBIE, 2018.

FRANÇA, Carlos R.; SILVA, Tatiana da. A utilização da Realidade Virtual e Aumentada no Ensino de Ciências no Brasil. [2017?], 18f, Programa de Pós-Graduação em Educação Científica e Tecnológica - Universidade Federal de Santa Catarina (UFSC).

GREIS, Luciano Kercher; REATEGUI, Eliseo. Um Simulador Educacional para Disciplina de Física em Mundos Virtuais. Renote: Revista Novas Tecnologias na Educação, Porto Alegre, v. 8, n. 2, p.1-10, jul. 2010.

O'KANE, James F.; TAYLOR R.; SPENCELEY J.R. Simulation as an essential tool for advanced manufacturing technology problems. Journal Of Materials Processing Technology. [s. L.], v.107, p. 412-424. nov. 2000.

KIRNER, Claudio et al. Fundamentos e Tecnologia de Realidade Virtual e Aumentada. Belém, PA: [s.n.], 2006.

KIRNER, Claudio; SISCOUTTO, Robson. Realidade Virtual e Aumentada: Conceitos, Projeto e Aplicações. Petrópolis, RJ: [s.n.], 2007.

ODUM, Eugene P. Ecologia. Rio de Janeiro: Guanabara Koogan. 1988.434 p.

REIS, Alessandro Vieira dos; GONÇALVES, Berenice dos Santos. Interfaces Tangíveis: Conceituação e Avaliação. Estudos em Design, Rio de Janeiro, v. 24, n. 2, p.92-111, 2016.

RICKLEFS, Robert E. A Economia da Natureza. 6. ed. Rio de Janeiro: Guanabara Koogan, 2010. $572 \mathrm{p}$. 
VIII Congresso Brasileiro de Informática na Educação (CBIE 2019)

Anais do XXV Workshop de Informática na Escola (WIE 2019)

ROMÃO, Viviane Pellizzon Agudo; GONÇALVES, Marília Matos. Realidade Aumentada: Conceitos e Design. Unoesc \& Ciência, Joaçaba, v.4, n.1, p.23-34, 2013.

TORGA, Bruno Lopes Mendes. Modelagem, Simulação e Otimização em Sistemas Puxados de Manufatura. 2007. 152 f. Dissertação (Mestrado) - Curso de Engenharia de Produção, Universidade Federal de Itajubá, Itajubá, 2007.

ULLMER, Brygg; ISHII, Hiroshi. Emerging frameworks for tangible user interfaces. In: CARROL, John M. (Ed.). Human-Computer Interaction in the New Millennium. Ann Arbor, MI, U.S.A: University of Michigan. Ann Arbor, 2001. p. 579-601.

VIGLIO, José Eduardo; FERREIRA, Lúcia da Costa. O conceito de ecossistema, a ideia de equilíbrio e o movimento ambientalista. Caderno Eletrônico de Ciências Sociais, Vitória, v. 1, n. 1, p.1-17, 2013. 